\title{
Fenómeno de Raynaud do mamilo em mulheres a amamentar: relato de três casos clínicos
}

Arnaldo Abrantes, ${ }^{1}$ Dusan Djokovic, ${ }^{2}$ Catarina Bastos, ${ }^{3}$ Patrizia Veca ${ }^{4}$

\section{RESUMO}

Introdução: O fenómeno de Raynaud resulta de vasoespasmo recorrente nas extremidades do corpo em resposta ao frio ou stress emocional. Manifesta-se clinicamente com dor, parestesias e alteração da cor da pele, afectando mais frequentemente os dedos das mãos e pés. O fenómeno de Raynaud do mamilo é uma causa subestimada de dor mamilar e abandono de aleitamento materno.

Caso 1: Mulher de 39 anos, G2P1 (parto pré-termo eutócico), com antecedentes pessoais de lúpus eritematoso sistémico, iniciou episódios de dor mamilar bilateral intensa desde a segunda semana pós-parto, associada a alteração trifásica da coloração do mamilo durante e após a amamentação. O diagnóstico do fenómeno de Raynaud foi estabelecido com base na história clínica e após a realização de teste de provocação com reprodução imediata de sintomas e sinais. A doente foi medicada com nifedipina, tornando-se assintomática após quatro semanas de tratamento.

Caso 2: Doente de 30 anos, G1P1 (parto de termo eutócico), com antecedentes pessoais irrelevantes, foi observada por episódio doloroso provocado por amamentação no seio direito, caracterizados ainda por palidez mamilar, cianose e hiperemia subsequentes. A senhora recusou tratamento farmacológico. No entanto, a utilização de bombas de esvaziamento mamário resultou em resolução de sintomatologia mamila descrita.

Caso 3: Mulher de 31 anos, G1P1 (parto de termo distócico com aplicação de ventosa), fumadora, com alergias alimentares e ao pólen, sem outros antecedentes pessoais relevantes, iniciou dor mamilar bilateral, intensa e intermitente, provocada por amamentação e acompanhada com alteração trifásica da coloração mamilar. Por sintomatologia altamente incapacitante, sem alívio significativo com cuidados locais propostos, suspendeu a amamentação antes de tentativa de tratamento farmacológico. Comentário: O fenómeno de Raynaud do mamilo é uma causa tratável de dor durante a amamentação. Sendo a causa comum do abandono da amamentação, deve ser reconhecido e tratado precocemente.

Palavras-chave: Fenómeno de Raynaud; Dor Mamilar; Amamentação.

\section{INTRODUÇÃO}

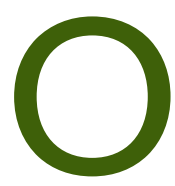

fenómeno de Raynaud (FR) foi descrito pela primeira vez em 1862 por Maurice Raynaud como um vasoespasmo intermitente dos pequenos vasos do sistema arterial, afectando principalmente as arteríolas nos dedos de mãos e pés. ${ }^{1}$ Hoje é considerado frequente, com prevalência global de 3-12,5\% em homens, ${ }^{2} 6-20 \%$ em mulheres $^{2} \mathrm{e}$ mais de $20 \%$ na subpopulação feminina em idade fértil. ${ }^{3}$ Apesar de manifestar-se mais tipicamente nas extremidades dos membros superiores e inferiores, o FR observa-se com frequência variável em outras regiões anatómicas, incluindo nariz, ouvidos, mamilo, circulação coronária ou gastrointestinal, pénis e placenta. ${ }^{1,4} \mathrm{O}$ vasoespasmo das arteríolas causa isquemia transitória, clinicamente manifestada por palidez cutânea, sendo

${ }^{1}$ Médico interno de Medicina Geral e Familiar - Unidade de Saúde Familiar do Dafundo - ACES Oeiras e Lisboa Ocidental

'Médico interno de Ginecologia e Obstetrícia, Doutor em Biomedicina, Hospital São Francisco e Xavier, Centro Hospitalar de Lisboa Ocidental

${ }^{3}$ Médica interna de Medicina Geral e Familiar - Unidade de Saúde Familiar da Sobreda - ACES Almada e Seixal

${ }^{4}$ Médica especialista de Ginecologia e Obstetrícia - Hospital São Francisco e Xavier, Centro Hospitalar de Lisboa Ocidental 
seguida por cianose da região afectada (desoxigenação do sangue venoso) e rubor/eritema subsequente (vasodilatação reflexa). ${ }^{1}$ A alteração trifásica da coloração da pele é usualmente associada a dor, sensação de queimadura ou outras parestesias. O vasoespasmo é mais comumente desencadeado por temperaturas ambientais baixas, mas outros eventos precipitantes foram relatados, nomeadamente stress emocional. ${ }^{5}$

De acordo com vários estudos, este fenómeno doloroso representa uma causa importante do abandono da amamentação, ${ }^{1,3,6}$ que oferece benefícios comprovados ao nível da nutrição e protecção imunológica do lactente e reforça a ligação mãe-filho.$^{6-7} \mathrm{~A}$ hipogalactia surge como o principal motivo, seguida da dor mamilar como segunda causa mais frequente de abandono precoce da amamentação. Até $96 \%$ das puérperas que amamentam experienciam dor mamilar e, por consequência, uma percentagem significativa irá substituir a amamentação pelo biberão. ${ }^{8-9}$ As principais causas de dor mamilar são: ingurgitamento mamário fisiológico ou patológico, dermatite atópica, dermatite alérgica ou de contacto, trauma mamilar, infecção mamilar secundária a Staphilococcus aureus ou a Candida albicans e o FR mamilar' (Figura 1).

O FR mamilar é uma entidade patológica subdiagnosticada. Geralmente não é reconhecido por médicos assistentes e, portanto, não especificamente tratado, embora a medicação com nifedipina possa proporcionar um controlo favorável dos sintomas. Com o presente artigo apresentamos três casos de FR do mamilo em mulheres a amamentar.

\section{CASO CLÍNICO 1}

Identificação: D.T., sexo feminino, 39 anos, natural da Sérvia, a viver em Portugal desde há dois anos. Licenciada, actualmente desempregada, vive em união de facto com o companheiro em casa própria. Trata-se de uma família nuclear, de classe média de Graffare no estádio II do Ciclo Duvall.

Antecedentes obstétricos: Gesta 2 para 1 (interrupção de gravidez voluntária com curetagem uterina, sem intercorrências, aos 25 anos; gestação evolutiva sem complicações, concebida espontaneamente aos 38 anos de idade, com parto pré-termo eutócico às 35 semanas e cinco dias).

Antecedentes pessoais: Diagnosticada aos 24 anos com lúpus eritematoso sistémico (LES), com seguimento em consulta de reumatologia, medicada com prednisolona (10mg $1 \mathrm{x} /$ dia) e sulfato de hidroxicloroquina (200mg 1x/dia), apresentando-se clinicamente estável. Sem outras patologias conhecidas.

Antecedentes familiares: Irrelevantes.

Doença actual: Um mês depois do parto, a doente recorreu ao serviço de urgência obstétrica/ginecológi-

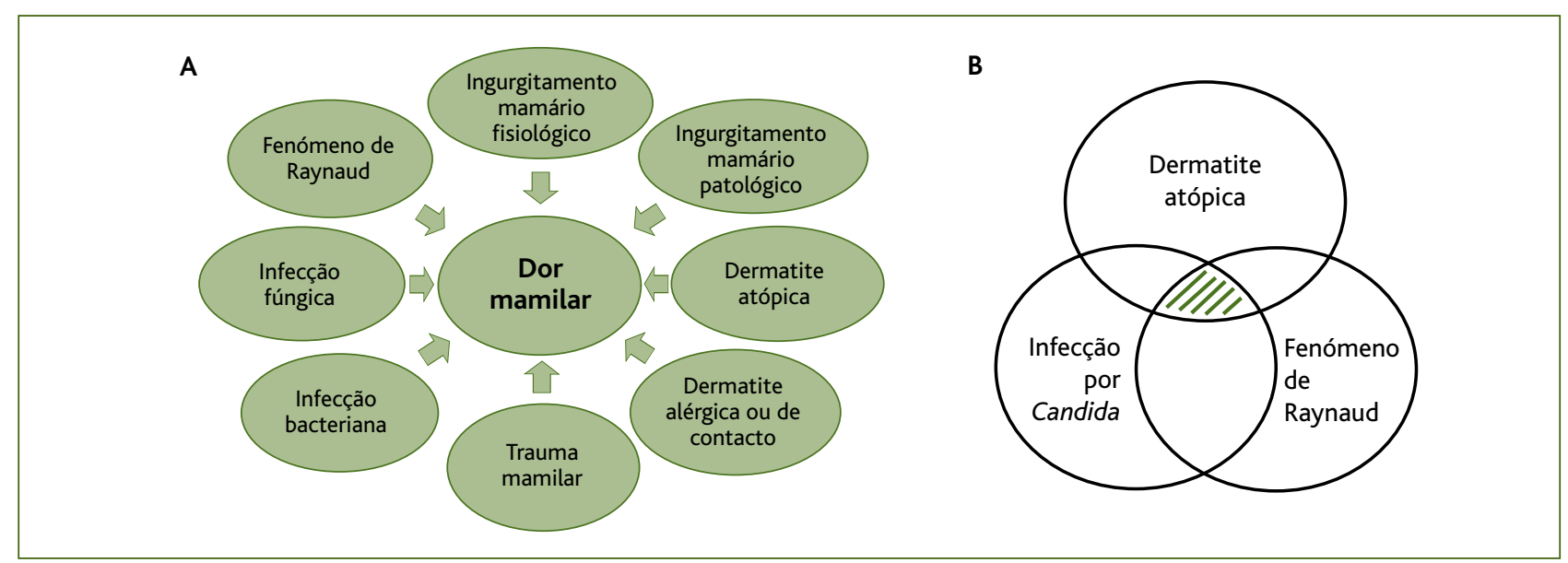

Figura 1. Etiologia da dor mamilar. (A) Causas principais da dor mamilar em mulheres a amamentar. (B) Diagrama de Venn ilustrando o conceito da etiologia multifactorial da dor mamilar (e.g., dermatite atópica, infecção por Candida albicans e fenómeno de Raynaud); este tipo de apresentação gráfica pode ser uma ferramenta importante para elucidar e encorajar as doentes na situação de insucesso terapêutico inicial e/ou nos casos de ser necessário combinar várias terapias. 

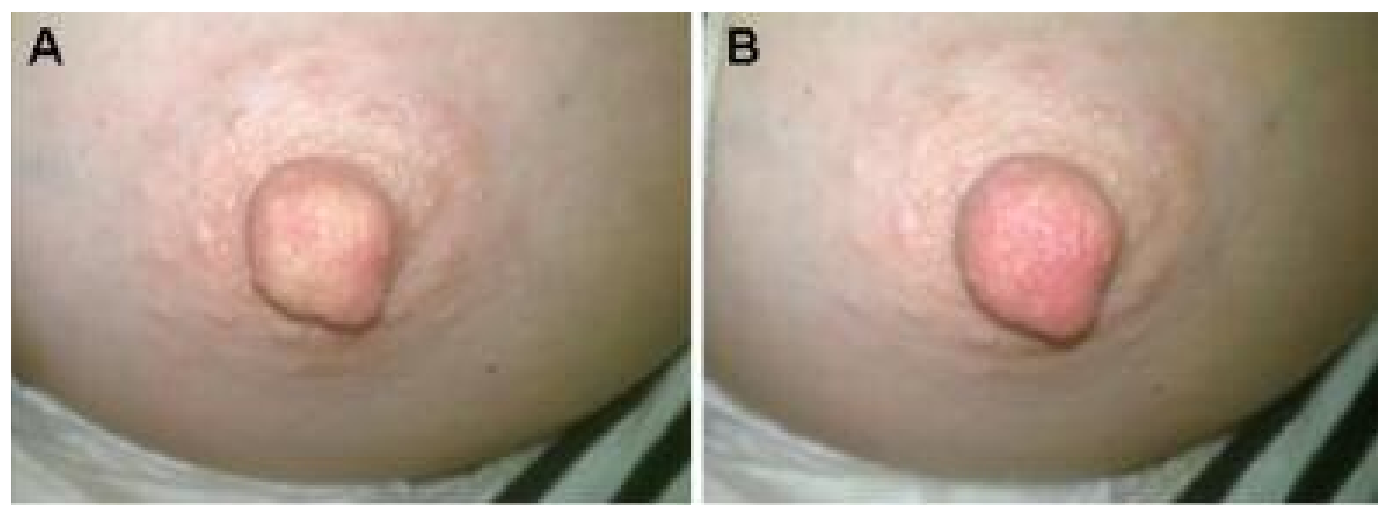

Figura 2. Teste de provocação com compressa de água fria (caso clínico 1). (A) Fase isquémica clinicamente manifestada por dor e palidez do mamilo. (B) Fase de vasodilatação reflexa caracterizada por rubor mamilar.

ca por episódios de dor mamilar bilateral intensa desde a segunda semana pós-parto, associada a alteração trifásica da coloração do mamilo (branco-azul-vermelho) durante e após a amamentação. Referiu como factor de agravamento os locais frios e de alívio os locais quentes. Estava a realizar aleitamento materno exclusivo, mas tencionava abandonar a amamentação por intensas queixas álgicas. Por incapacidade de observar o mamilo durante a amamentação (recorreu sem ser acompanhada por lactente) e ainda a não variabilidade de temperatura no gabinete médico, procedeu-se a um teste de provocação, através da colocação de uma compressa humidificada com água fria sobre o mamilo. Durante a realização do teste houve o desencadear da dor, embora com menor intensidade e, no instante após a remoção da compressa, observou-se descoloração (palidez) do mamilo, seguida de hiperemia (Figura 2). Foi medicada com nifedipina $5 \mathrm{mg} 3 \mathrm{x} /$ dia durante uma semana e posteriormente $1 \mathrm{x} /$ dia. Apresentou boa resposta, com redução da frequência das "crises" e sem queixas após quatro semanas de tratamento.

\section{CASO CLÍNICO 2}

Identificação: A.M., mulher de 30 anos, portuguesa, licenciada, pertence a uma família nuclear, de classe média de Graffar e no estádio II do Ciclo Duvall.

Antecedentes obstétricos: Gesta 1 para 1 (gravidez espontânea aos 30 anos, sem intercorrências, com parto de termo eutócico dentro de água às 40 semanas).

Antecedentes pessoais e familiares: Irrelevantes.

Doença actual: Depois de divulgação do caso clínico
1 entre os colegas e colaboradores do Hospital de São Francisco Xavier/ACES Oeiras e Lisboa Ocidental, foi referenciada esta doente que apresentava episódios de dor e descoloração do mamilo direito desde o segundo mês pós-parto. A doente associou o início de queixas com o início de época mais fria do ano. Observou-se um episódio doloroso provocado por amamentação no seio direito, caracterizado por esbranquecimento cutâneo mamilar que se mantinha durante quase uma hora, sendo seguido por curtas fases de cianose e hiperemia. A senhora recusou tratamento farmacológico. Optou por utilização de bombas de esvaziamento mamário, que resultou em franca redução e, subsequentemente, em desaparecimento de sintomatologia mamilar referida.

\section{CASO CLÍNICO 3}

Identificação: R.G., sexo feminino, 31 anos, portuguesa. Completou o ensino secundário, desempregada, pertence a uma família de classe média-baixa de Graffar e no estádio II do Ciclo Duvall.

Antecedentes obstétricos: Gesta 1 para 1 (gravidez espontânea sem complicações, concebida aos 30 anos de idade, com parto de termo distócico às 39 semanas e dois dias - aplicação de ventosa por paragem de trabalho de parto no período expulsivo).

Antecedentes pessoais: Alergias alimentares (marisco) e ao pólen. Hábitos tabágicos (10-15 cigarros/ /dia). Sem outros antecedentes pessoais relevantes.

Antecedentes familiares: Irrelevantes.

Doença actual: Iniciou amamentação já no bloco de partos, com boa adaptação do recém-nascido ao pei- 
to. Sem queixas relevantes até à sexta semana pós-parto, altura em que inicia dor mamilar bilateral, intensa e intermitente (durante e até 15 minutos depois de amamentação). Não se conseguiram identificar factores específicos de agravamento ou de alívio. De acordo com a informação clínica do médico que referenciou a doente um mês após o início de sintomas, as queixas álgicas coincidiam com alteração trifásica da coloração mamilar (palidez-cianose-rubor). No entanto, por considerar a sintomatologia altamente incapacitante, sem alívio significativo com medidas terapêuticas propostas (aquecimento, utilização de bombas eléctricas de esvaziamento), suspendeu a amamentação antes de ser observada pela equipa. Apresentou-se na consulta assintomática, com exame mamário sem alterações.

\section{COMENTÁRIO}

Mulheres em idade fértil apresentam um risco aumentado de desenvolver o FR devido, pelo menos parcialmente, à resposta vasomotora exagerada associada à elevação dos níveis de estrogénios. ${ }^{1}$ Os estrogénios aumentam a resposta do músculo liso à activação dos $\alpha 2 c$-adrenoreceptores que, associado à exposição ao frio, induz a vasoconstrição intensa das artérias e arteríolas cutâneas. O stress emocional provoca a libertação de norepinefrina pelo sistema nervoso simpático, que se liga aos receptores adrenérgicos vasculares sobre-regulados por estrogénios, podendo também desencadear o FR. De facto, o próprio ato de amamentação é considerado como situação de stress emocional por um significativo número de puérperas. ${ }^{1}$

Apesar de haver poucos casos a relatar o FR do mamilo em mães a amamentar, uma revisão retrospectiva $^{1}$ realizada por Barrett e colaboradores em 2013, incluiu 22 casos, utilizando critérios diagnósticos propostos para o FR do mamilo [episódios de dor mamilar moderada/intensa associada à amamentação e $\geq$ dois dos seguintes critérios: 1) alteração trifásica/bifásica da cor do mamilo, 2) sensibilidade ao frio ou alterações da cor dos mãos/pés com o frio, 3) ausência de resposta ao tratamento com antifúngicos orais (Figura 3)]. Maioria das mulheres incluídas $[n=17(77 \%)]$ iniciou os episódios da dor mamilar nas primeiras duas semanas pós-parto. Todas referiram dor associada à amamentação, sendo que cinco (28\%) referiram aumento da dor no início da amamentação e 13 (72\%) experienciaram dor antes, durante e após a amamentação. A dor foi ainda descrita como moderada a severa, do tipo pontada ou facada. Relativamente aos presentes casos, as três mulheres referiram dor mamilar moderada a intensa, provocada pelo acto da amamentação e com alterações bifásicas ou trifásicas da coloração do mamilo. Além disso, duas das três mulheres reportaram agravamento da dor nos locais frios. Em todos os casos, rejeito-se clinicamente a hipótese de micose por sintomatologia estritamente intermitente, sem prurido, descamação ou outras alterações patognomónicas.

A anamnese e o exame objectivo são essenciais para diagnóstico do FR mamilar. A informação confirmando existência de um tratamento antifúngico oral prévio, completo e sem sucesso é valiosa, aumentando a probabilidade de tratar-se do FR. No entanto, nenhuma das três mulheres observadas apresentava um tratamento prévio com antifúngico e, no caso de duas delas, o exame objectivo era aparentemente dentro da normalidade. Assim, foi efectuado um teste de provocação (caso clínico 1) com uma compressa de água fria, que foi positivo, pois surgiu dor mamilar (embora menos intensa que habitual) e alteração bifásica da coloração do mamilo logo depois da remoção da compressa (Figura 2). No segundo caso, com exame físico aparentemente sem alterações (caso clínico 3), não foi realizado o teste de provocação por senhora já ter desistido da amamentação. Pretende-se avaliar este teste de provocação nos futuros casos de suspeita de FR como um método diagnóstico complementar.

Como já referido na introdução, a dor mamilar tem várias etiologias e representa um risco para o abandono precoce da amamentação, sendo por isso fundamental realizar um diagnóstico precoce, correcto e tratamento imediato. O ingurgitamento mamário patológico ocorre mais frequentemente no $3 .^{\circ}-5 .^{\circ}$ dia pós-parto, sendo caracterizado por um aumento de volume da mama, dor e áreas difusas avermelhadas, edemaciadas e brilhantes. ${ }^{10}$ Os mamilos tornam-se achatados, o que dificulta a pega do lactente, piorando ainda mais o ingurgitamento (círculo vicioso). Uma dermatite atópica mamilar pode cursar com descamação e exsudação da auréola e mamilo, além do prurido habitual. O tratamento depende da etiologia e em casos simples deverá resolver os sintomas em menos de uma semana. Trauma mamilar por posicionamento e pega 


\section{DOR MAMILAR}

(moderada a intensa, crónica - geralmente com duração de $\geq 4$ semanas)

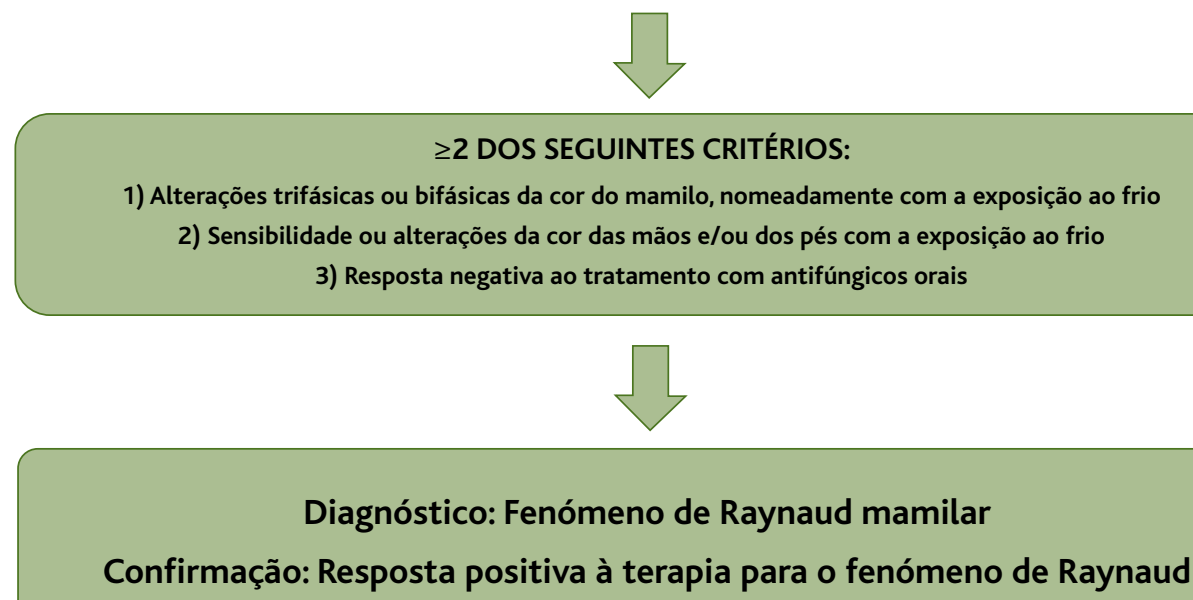

Confirmação: Resposta positiva à terapia para o fenómeno de Raynaud

Figura 3. Critérios de diagnóstico para o fenómeno de Raynaud mamilar propostos por Barrett e seus colaboradores. ${ }^{1}$

inadequada do lactente é outra causa frequente de dor mamilar. O risco de traumatismo está nomeadamente aumentado em situações como mamilos curtos/planos ou invertidos, disfunções orais da criança, freio da língua excessivamente curto, sucção não-nutritiva prolongada e uso incorrecto de bombas de extracção de leite.$^{10}$ Finalmente, a dor rubor, edema e mamilar, podem ter etiologia infecciosa. A infecção causada por Staphilococcus aureus geralmente ocorre sobre um mamilo lesionado, enquanto a infecção puerperal da mama por Candida albicans costuma ocorrer na presença de mamilos húmidos e com lacerações, podendo ser superficial ou atingir ductos lactíferos. O uso de antibióticos prévios, contraceptivos orais, esteróides, candidíase vaginal ou o uso de chupeta contaminada aumenta o risco de candidíase. A infecção mamilar fúngica manifesta-se tipicamente por prurido e dor nos mamilos, tipo queimadura ou picadas, persistente após as mamadas. O exame físico costuma revelar os mamilos edemaciados, eritematosos e brilhantes. O lactente frequentemente apresenta crostas brancas orais que devem ser distinguidas das crostas de leite. ${ }^{10}$ É de importância fundamental destacar que, em muitos casos, a dor mami- lar tem uma etiologia multifactorial. ${ }^{11}$ Por exemplo, num doente com dermatite atópica pode-se paralelamente desenvolver o FR e ainda instalar infecção a Candida albicans (Figura 1B). O tratamento deve ser dirigido a todas as causas presentes. De acordo com Heller e seus colaboradores, ${ }^{11}$ o estabelecimento do diagnóstico do FR não é, na maioria das vezes, razão para excluir outras causas de dor mamilar, embora o fenómeno possa representar o factor que contribui predominantemente às queixas álgicas da doente. Por outo lado, se o tratamento antimicrobiano e/ou terapêutica de dermatite atópica não melhoram o quadro clínico de dor e intolerância ao frio, deve-se considerar o FR mamilar como um mecanismo fisiopatológico concomitante.

Aquando do diagnóstico correcto de FR mamilar, as doentes com dor mamilar podem melhorar notavelmente se aconselhadas a evitar temperaturas frias, usar técnicas para manter as mamas e mamilos quentes e evitar substâncias vasoconstritoras como a cafeína e nicotina. ${ }^{1}$ A nifedipina, um antagonista dos canais de cálcio aprovado pela Academia Americana de Pediatria para o uso materno durante a amamentação, funciona 
como vasodilatador e representa a farmacoterapia de primeira linha para o FR mamilar..$^{12-13}$ No estudo retrospectivo de Barrett, ${ }^{1}$ a medicação com nifedipina melhorou significativamente os sintomas do FR mamilar e foi, em geral, bem tolerada. Especificamente, 10 em 15 mulheres (67\%) medicadas com este vasodilatador reportaram uma diminuição ou resolução da dor mamilar, como a doente tratada com nifedipina (caso clínico 1). Das outras cinco mulheres, duas não notaram melhorias, enquanto três mulheres interromperam o tratamento por surgimento dos efeitos secundários. No entanto, estas mulheres apresentaram melhoria sintomática evitando o uso de substâncias vasoconstritoras e com cuidados locais, incluindo as técnicas de aquecimento.

Recomenda-se prescrição de uma formulação de libertação lenta de nifedipina com posologia de 30$-60 \mathrm{mg} / \mathrm{dia}$. O tratamento pode durar de duas semanas até ao fim do período de amamentação. A medicação com nifedipina exige que as doentes sejam avisadas sobre os efeitos adversos deste medicamento, nomeadamente náuseas, hipotensão, taquicardia, cefaleias e tonturas. Se a doente experienciar algum efeito lateral, recomenda-se redução da dose diária até 10mg. Adicionalmente, as doentes devem ser tranquilizadas relativamente à segurança desta modalidade terapêutica. A nifedipina apenas passa para o leite materno em baixas doses, $<5 \%$ da dose materna. ${ }^{14}$

Resumindo, o FR do mamilo deve ser considerado em mulheres que se apresentam com dor mamilar durante a amamentação. O diagnóstico pode ser facilitado utilizando os critérios propostos por Barrett e seus colaboradores. $\mathrm{O}$ reconhecimento precoce desta entidade patológica e tratamento adequado, que se baseia nos cuidados locais, evicção de vasoconstritores e farmacoterapia com nifedipina, podem ajudar a prevenir o abandono da amamentação devido às queixas álgicas.

\section{AGRADECIMENTO}

Agradecemos a colaboração da Sra. Enfermeira Maria de Fátima Cardoso Esteves (Unidade de Cuidados na Comunidade - ACES Lisboa Ocidental e Oeiras) no recrutamento das puérperas a amamentar para eventual inclusão nesta apresentação de casos clínicos.

\section{REFERÊNCIAS BIBLIOGRÁFICAS}

1. Barrett ME, Heller MM, Stone HF, Murase JE. Raynaud phenomenon of the nipple in breastfeeding mothers: an underdiagnosed cause of nipple pain. JAMA Dermatol. 2013;149(3):300-6.

2. Goundry B, Bell L, Langtree M, Moorthy A. Diagnosis and management of Raynaud's phenomenon. BMJ. 2012;344:e289.

3. Lawlor-Smith L, Lawlor-Smith C. Vasospasm of the nipple-a manifestation of Raynaud's phenomenon: case reports. BMJ. 1997;314 (7081):644-5.

4. Morino C, Winn SM. Raynaud's phenomenon of the nipples: an elusive diagnosis. J Hum Lact. 2007;23(2):191-3.

5. Flavahan NA. A vascular mechanistic approach to understanding Raynaud phenomenon. Nat Rev Rheumatol. 2015;11(3):146-58.

6. Anderson JE, Held N, Wright K. Raynaud's phenomenon of the nipple: a treatable cause of painful breastfeeding. Pediatrics. 2004;113(4): e360-4.

7. Neville MC, Anderson SM, MCManaman JL, Badger TM, Bunik M, Contractor $\mathrm{N}$, et al. Lactation and neonatal nutrition: defining and refining the critical questions. J Mammary Gland Biol Neoplasia. 2012;17(2): 167-88.

8. Morland-Schultz K, Hill PD. Prevention of and therapies for nipple pain: a systematic review. J Obstet Gynecol Neonatal Nurs. 2005;34(4):42837.

9. Ziemer MM, Paone JP, Schupay J, Cole E. Methods to prevent and manage nipple pain in breastfeeding women. West J Nurs Res. 1990;12(6): 732-43.

10. Giugliani ER. [Common problems during lactation and their management]. J Pediatr (Rio J). 2004;80(5 Suppl):S147-54.

11. Heller MM, Fullerton-Stone H, Murase JE. Caring for new mothers: diagnosis, management and treatment of nipple dermatitis in breastfeeding mothers. Int J Dermatol. 2012;51(10):1149-61.

12. Garrison CP. Nipple vasospasms, Raynaud's syndrome, and nifedipine. J Hum Lact. 2002;18(4):382-5.

13. Wu M, Chason R, Wong M. Raynaud's phenomenon of the nipple. Obstet Gynecol. 2012;119(2 Pt 2):447-9.

14. Kinlay JR, O'Connell DL, Kinlay S. Incidence of mastitis in breastfeeding women during the six months after delivery: a prospective cohort study. Med J Aust. 1998;169(6):310-2.

\section{CONFLITO DE INTERESSES}

Os autores declaram não ter conflitos de interesses.

As doentes deram o seu consentimento para a publicação de este artigo.

\section{ENDEREÇO PARA CORRESPONDÊNCIA}

Dusan Djokovic

Av. Defensores de Chaves, 75 - 3. ${ }^{\circ}-1000-114$ Lisboa

E-mail: dusan.d.djokovic@gmail.com

Recebido em 10-06-2015

Aceite para publicação em 09-02-2016 


\section{ABSTRACT}

\section{RAYNAUD'S PHENOMENON OF THE NIPPLE IN BREASTFEEDING WOMEN: A REPORT OF THREE CASES}

Introduction: Raynaud's phenomenon results from recurrent vasospasm in acral areas of the body in response to cold or emotional stress. It is characterized by pain, paresthesia, and skin colour changes, affecting most frequently the fingers and toes. Raynaud's phenomenon is an underdiagnosed cause of nipple pain and cessation of breastfeeding.

Case 1: A 39-year-old woman, G2P1 with a eutocic preterm delivery and a history of systemic lupus erythematosus, complained of episodes of intense bilateral nipple pain from the second postpartum week. The pain was associated with a triphasic colour change of the skin of the nipple skin during and after breastfeeding. The diagnosis of Raynaud's phenomenon was made based on the history and after the performance of a provocative test causing the prompt onset of the symptoms and signs. The patient began treatment with nifedipine and was asymptomatic within 4 weeks.

Case 2: A 30-year-old patient, G1P1 with a eutocic delivery at term, with no previous medical history, was seen because of episodes of pain in the right breast caused by breastfeeding. They were accompanied by blanching of the skin of the nipple with subsequent cyanosis followed by hyperemia. The patient refused medication. The use of an electric breast electric pump resulted in resolution of her symptoms.

Case 3: A 30-year-old women, G1P1, had a dystocic delivery at term requiring application of the vacuum extractor. She was a smoker and had food and pollen allergies but no other relevant medical history. She presented with severe, bilateral, intermittent nipple pain caused by breastfeeding and accompanied by a triphasic change in nipple colour. Due to her disabling symptoms and without significant relief from local nipple care, she stopped breastfeeding before trying pharmacological treatment. Comment: Raynaud's phenomenon of the nipple is a treatable cause of pain during breastfeeding. It should be recognized and treated as early as possible to prevent cessation of breastfeeding.

Keywords: Raynaud's Phenomenon; Nipple Pain; Breastfeeding. 